OPEN ACCESS

Edited by:

Naoki Osada,

Hokkaido University, Japan

Reviewed by:

Jian-Feng Mao,

Beijing Forestry University, China

Jacob A. Tennessen,

Oregon State University, USA

${ }^{*}$ Correspondence:

Renchao Zhou

zhrench@mail.sysu.edu.cn

Specialty section:

This article was submitted to Evolutionary and Population Genetics, a section of the journal

Frontiers in Plant Science

Received: 28 March 2017

Accepted: 12 April 2017

Published: 26 April 2017

Citation:

Zou P, Ng WL, Wu W, Dai S, Ning Z,

Wang S, Liu Y, Fan Q and Zhou R

(2017) Similar Morphologies but Different Origins: Hybrid Status of Two

More Semi-creeping Taxa

of Melastoma. Front. Plant Sci. 8:673.

doi: 10.3389/fpls.2017.00673

\section{Similar Morphologies but Different Origins: Hybrid Status of Two More Semi-creeping Taxa of Melastoma}

\author{
Peishan Zou ${ }^{1}$, Wei Lun $\mathrm{Ng}^{1}$, Wei Wu${ }^{1}$, Seping Dai ${ }^{2}$, Zulin Ning ${ }^{3}$, Shuqiong Wang ${ }^{1}$, \\ Ying Liu', Qiang Fan ${ }^{1}$ and Renchao Zhou ${ }^{1 *}$
}

${ }^{1}$ State Key Laboratory of Biocontrol and Guangdong Provincial Key Laboratory of Plant Resources, School of Life Sciences, Sun Yat-sen University, Guangzhou, China, ${ }^{2}$ Guangzhou Institute of Forestry and Landscape Architecture, Guangzhou, China, ${ }^{3}$ Guangdong Provincial Key Laboratory of Applied Botany, South China Botanical Garden, Chinese Academy of Sciences, Guangzhou, China

Inferring the origins of hybrid taxa based on morphology alone is difficult because morphologically similar hybrids can arise from hybridization between different populations of the same parental species or be produced by hybridization of different parental species. In this study, we investigated the origins of two semi-creeping taxa in Melastoma, which are morphologically similar to a natural hybrid, $M$. intermedium, by sequencing a chloroplast intergenic spacer, nuclear ribosomal internal transcribed spacer and two low-copy nuclear genes (tpi and cam) in these taxa and their putative parental species. Our sequence analysis provides compelling evidence for the hybrid status of the two semi-creeping taxa: one originating from hybridization between M. dodecandrum and M. malabathricum, and the other between M. dodecandrum and $M$. normale. The origins of these hybrids are therefore clearly different from $M$. intermedium, and morphological similarity for the three hybrids is most likely due to their origins from hybridization between the same creeping species $M$. dodecandrum and a different erect species in each of the three cases. We also observed low rate of introgression from $M$. normale to $M$. dodecandrum, and genetic exchange between them may transfer adaptive traits to $M$. dodecandrum. Rare occurrence of these two hybrids may be due to small range overlaps between parental species in one case, and different flowering periods between parental species in the other.

Keywords: natural hybridization, Melastoma, molecular analysis, introgression, nuclear genes

\section{INTRODUCTION}

Natural hybridization is pervasive in flowering plants and it can generate evolutionary novelty through the formation of hybrid lineages or interspecific introgression (Arnold, 1997; Rieseberg et al., 1998; Goulet et al., 2017). When one species hybridizes with another, hybrids can establish locally and then may disperse to other locations where one or both parental species do not occur (e.g., Fitzpatrick et al., 2010). If parental species overlap in geographic distribution in multiple locations, hybrids may arise independently from hybridization between local populations of parental species (e.g., Zhang et al., 2013). Under this circumstance, hybrids from different locations may show subtle or obvious morphological differences. This can be caused by morphological divergence in one or two parental species from different locations, or environmental differences 
between different locations. On the other hand, when one species hybridizes with two or more species, hybrids produced from different combinations of parental species may also show subtle or obvious morphological differences. Thus, different hybrid taxa with slight morphological dissimilarity may have arose from hybridization of the same parental species in different locations or hybridizations involving different combinations of parental species. Therefore, inferring the origins of such hybrid taxa based on morphology alone is difficult. In such cases, molecular means have been proven successful in determining the origins of various hybrid taxa (Zhang et al., 2013; Harrison and Larson, 2014).

Melastoma, a shrub genus distributed in tropical Asia and Oceania, has undergone rapid adaptive radiation, with more than 20 species diversified during the last one million years (Meyer, 2001; Renner and Meyer, 2001). Wong (2016), however, estimated that there are 80-90 species in this genus. Because there is substantial overlap in geographic distribution and flowering time between some species of this genus, interspecific hybridization is assumed to be relatively common (Dai et al., 2012; Liu et al., 2014). However, only two cases of natural hybridization have been confirmed using molecular means so far (Dai et al., 2012; Liu et al., 2014), and the role of natural hybridization in the evolution of this genus remains elusive.

Melastoma dodecandrum is the only creeping species in the genus, while the other species are all erect shrubs. Previously, we identified $M$. intermedium, a semi-creeping shrub, as a natural hybrid between $M$. dodecandrum and M. candidum (Dai et al., 2012). During our field survey of Melastoma in Guangdong in the past few years, we found two more semi-creeping taxa of Melastoma, one in Ruyuan and the other in Xinyi. Both taxa are of very rare occurrence. These two taxa have the similar stem type (i.e., semi-creeping) as $M$. intermedium, but there are subtle morphological differences between these three semicreeping taxa: the taxon found in Ruyuan and M. intermedium have very short appressed scales on the young twigs, while the taxon found in Xinyi has spreading bristles on the young twigs. The taxon found in Xinyi and $M$. intermedium has oblong leaves and strigose upper surfaces, while the taxon found in Ruyuan possesses lanceolate leaves and subglabrous upper surfaces (Supplementary Table S1). At the sampling site of Ruyuan, only $M$. dodecandrum and M. malabathricun coexist, while at the sampling site of Xinyi, only $M$. dodecandrum and $M$. normale co-occur. There was no $M$. candidum, one of the parental species of $M$. intermedium, at both sites.

The semi-creeping nature of the two newly found taxa results likely from separate hybridization events between the creeping M. dodecandrum and the erect M. malabathricum in Ruyuan, and M. normale in Xinyi. In this scenario, morphological differences between the three semi-creeping taxa may stem mainly from morphological differences among one of their parental species, M. candidum, M. malabathricun, and M. normale, since they share the other parental species, M. dodecandrum. However, there is also a possibility that these two taxa are in fact M. intermedium, germinated from seeds brought by birds from other locations where $M$. dodecandrum and $M$. candidum co-occur and hybridize. Birds have been known to be seed dispersers for Melastoma species (Whittaker and Jones, 1994;
Westcott et al., 2008). Morphological differences in this scenario may be caused by environmental differences.

Figuring out the status of a taxon in question is crucial for understanding its taxonomy and evolution. For this study, we collected samples from populations of the possible parental species $M$. dodecandrum, M. normale, M. malabathricum, and $M$. candidum, as well as the two unidentified taxa (designated as DM and DN hereafter, from Ruyuan and Xinyi, respectively). We sequenced one chloroplast intergenic spacer, nuclear ribosomal internal transcribed spacer (nrITS) and two low-copy nuclear genes ( $t p i$ and cam) to investigate the taxonomic status of the two newly found semi-creeping Melastoma taxa. Because introgression is a common consequence of hybridization and can contribute to transfer of adaptation (Arnold and Martin, 2009; Whitney et al., 2010), we also aimed to examine if there is any evidence of introgression between the putative parental species.

\section{MATERIALS AND METHODS}

\section{Plant Materials}

We sampled one population each of $M$. dodecandrum, M. malabathricum, and the unidentified taxon (DM) in Tianjingshan, Ruyuan, Guangdong, China, and one population each of $M$. dodecandrum, $M$. normale, and the other unidentified taxon (DN) in Dawuling, Xinyi, Guangdong. All three erect species occur in open habitats, while $M$. dodecandrum prefers slightly shady habitats. DM occurs along several trailsides near Tianjinshan, Ruyuan, with the elevations of 500-700 m. DN was found along the roadside in the Dawuling Nature Reserve, Xinyi, at an altitude of about 900-1000 m. It seems that both locations have experienced human disturbance due to road or trail constructions. Only four to five samples of the unidentified taxa were collected due to their scarcity in both locations. The sampling details are shown in Table 1. To test whether the two unidentified taxa are $M$. intermedium or not, we also collected samples of $M$. candidum from Ziyun Park, Longhai, Fujian, China. Morphological comparisons of these taxa are shown in Figure 1 and Supplementary Figures S1-S3 and Table S1. Leaves were sampled and dried in plastic bags with silica gel for subsequent DNA extraction.

\section{DNA Extraction, PCR, and Sequencing}

We extracted genomic DNA from dried leaf tissue using the CTAB method (Doyle and Doyle, 1987). We amplified the chloroplast $t r n \mathrm{~L}-\mathrm{trn \textrm {F }}$ intergenic spacer and nrITS regions using the universal primers trn-c and trn-f (Taberlet et al., 1991), and ITS4 and ITS5 (White et al., 1990), respectively. Two low-copy nuclear genes (tpi and cam), which encode triose phosphate isomerase and calmodulin, respectively, were PCR-amplified and sequenced using the primer sequences from Dai et al. (2012) and Chao et al. (2014), respectively. We purified the PCR products using the Pearl Gel Extraction Kit (Pearl Bio-tech, Guangzhou, China) and then directly sequenced them on an ABI 3730 DNA automated sequencer with the BigDye chemistry (Applied Biosystems, Foster City, CA, USA). For sequences with more than 
TABLE 1 | Sampling details of six taxa of Melastoma used in this study.

\begin{tabular}{|c|c|c|}
\hline Taxon & Locality & Sample siz \\
\hline M. dodecandrum $\left(\mathrm{D}_{\mathrm{R}}\right)$ & Tianjingshan, Ruyuan, Guangdong & 20 \\
\hline M. dodecandrum $\left(D_{x}\right)$ & Dawuling, Xinyi, Guangdong & 20 \\
\hline M. malabathricum (M) & Tianjinshan, Ruyuan, Guangdong & 18 \\
\hline M. normale $(\mathrm{N})$ & Dawuling, Xinyi, Guangdong & 20 \\
\hline M. candidum $(\mathrm{C})$ & Ziyun Park, Longhai, Fujian & 20 \\
\hline Putative hybrid between D and M (DM) & Tianjinshan, Ruyuan, Guangdong & 5 \\
\hline Putative hybrid between D and N (DN) & Dawuling, Xinyi, Guangdong & 4 \\
\hline
\end{tabular}

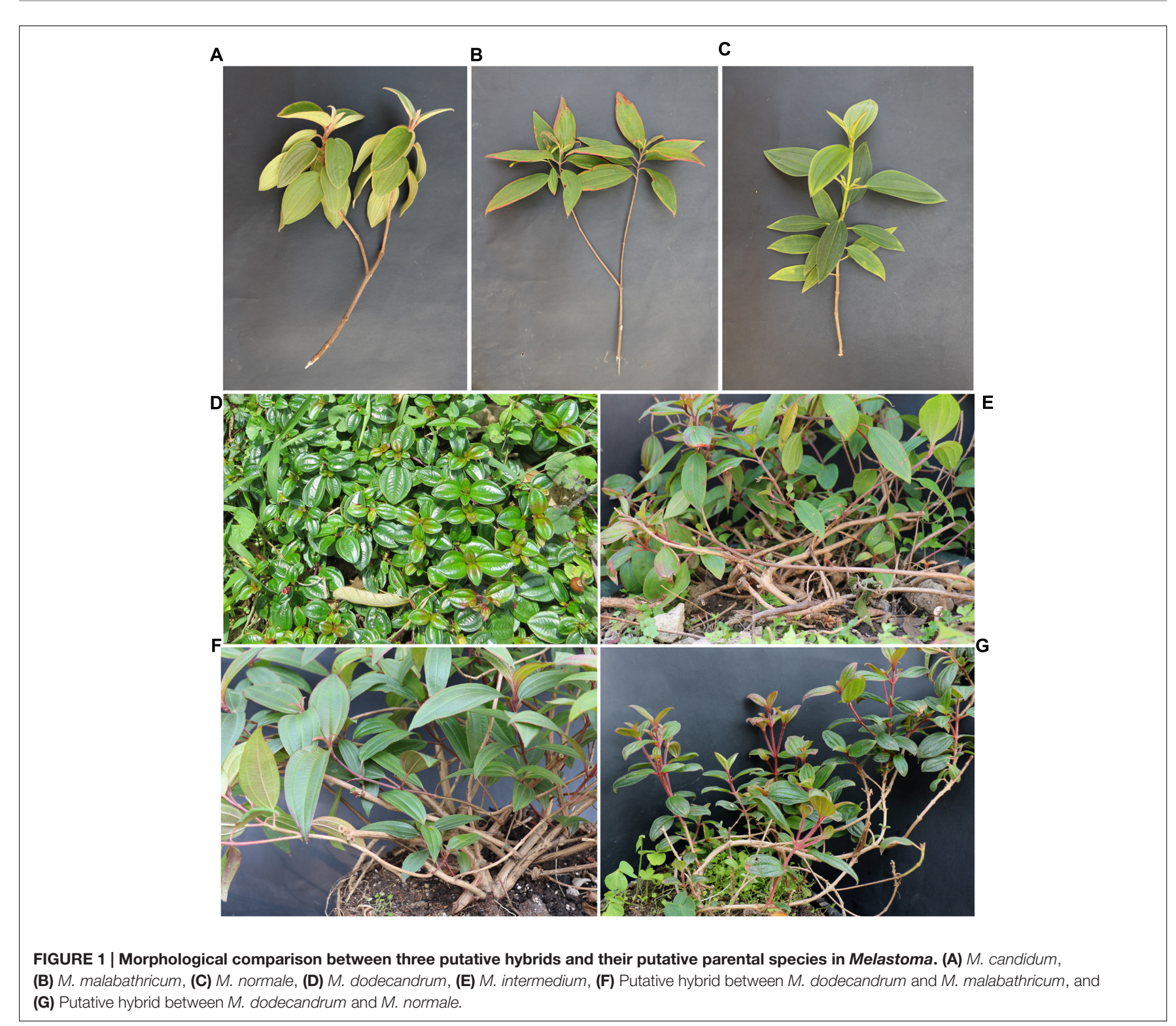

one polymorphic site and insertion/deletion polymorphisms, cloning-sequencing was conducted to phase the haplotypes. We conducted ligation reactions with a pMD18-T\&A cloning kit (Takara, Dalian, China) and selected eight positive colonies for each individual for sequencing. We deposited all the sequences in GenBank with accession numbers KY798014-KY798110.

\section{Sequence Analyses}

We edited the sequences using SeqMan (DNASTAR Inc., Madison, WI, USA) and aligned them using Clustal X (Thompson et al., 1997). The haplotypes of the nuclear loci that had no, or one, polymorphic site, were inferred using Phase (Librado and Rozas, 2009). We used the median-joining method 
TABLE 2 | Genotypes of the two hybrids of Melastoma at one chloroplast intergenic spacer (trnL-trnF), nrITS region, and two nuclear genes (tpi and cam).

\begin{tabular}{|c|c|c|c|c|c|}
\hline \multirow[t]{2}{*}{ Taxon } & \multirow[t]{2}{*}{ Sample ID } & \multicolumn{4}{|c|}{ Locus } \\
\hline & & trnF-trnL & nrITS & tpi & cam \\
\hline \multirow[t]{5}{*}{ The hybrid between M. dodecandrum and M. malabathricum (DM) } & DM1 & $\underline{\mathrm{T} 4}$ & $\underline{11}, \underline{\underline{13}}$ & $\underline{\mathrm{HR} 2}, \underline{\mathrm{HR} 4}$ & $\underline{\mathrm{HR} 2}, \mathrm{HR} 8$ \\
\hline & $\mathrm{DM} 2$ & $\overline{\mathrm{T} 1}$ & $\underline{11}, \underline{\underline{13}}$ & $\underline{\underline{\mathrm{HR} 1}}, \underline{\mathrm{HR} 3}$ & $\mathrm{HR} 4, \underline{\underline{\mathrm{HR}} 5}$ \\
\hline & DM3 & $\underline{\mathrm{T} 2}$ & $\underline{11}, \underline{\underline{13}}$ & $\underline{\underline{\mathrm{HR} 2}}, \underline{\mathrm{HR} 3}$ & $\mathrm{HR} 3, \overline{\mathrm{HR} 6}$ \\
\hline & DM4 & $\underline{\mathrm{T} 4}$ & $\underline{11}, \underline{\underline{13}}$ & $\underline{\mathrm{HR} 1}, \underline{\mathrm{HR} 3}$ & $\underline{\mathrm{HR}} 2, \mathrm{HR} 7$ \\
\hline & DM5 & $\overline{\overline{\mathrm{T} 4}}$ & $\underline{11}, \underline{\underline{13}}$ & $\underline{\underline{\mathrm{HR} 1}}, \underline{\mathrm{HR} 3}$ & $\underline{\mathrm{HR} 1}, \underline{\underline{\mathrm{HR} 5}}$ \\
\hline \multirow[t]{4}{*}{ The hybrid between M. dodecandrum and M. normale (DN) } & DN1 & T3 & $\underline{12}, \underline{13}$ & $\underline{\mathrm{HX} 2}, \mathrm{HX} 4$ & $\underline{H X 3}, \underline{H X 4}$ \\
\hline & DN2 & T3 & $\underline{12}, \underset{\sim}{13}$ & $\underline{H X 2}, \underline{H X 4}$ & $\underline{H X 3}, \underline{H X 4}$ \\
\hline & DN3 & T3 & $\underline{12}, \underline{13}$ & $\underline{H X 2}, \underline{H X}$ & $\underline{H X 1}, \underline{H X 4}$ \\
\hline & DN4 & T3 & 12,13 & $\underline{H X 1}, \underline{H X 3}$ & $\underline{H X 2}, \underline{H X} 4$ \\
\hline
\end{tabular}

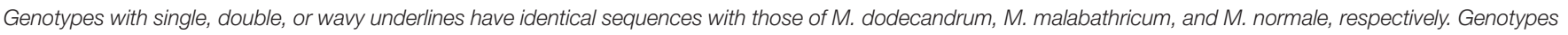
without underline are unique to hybrids.

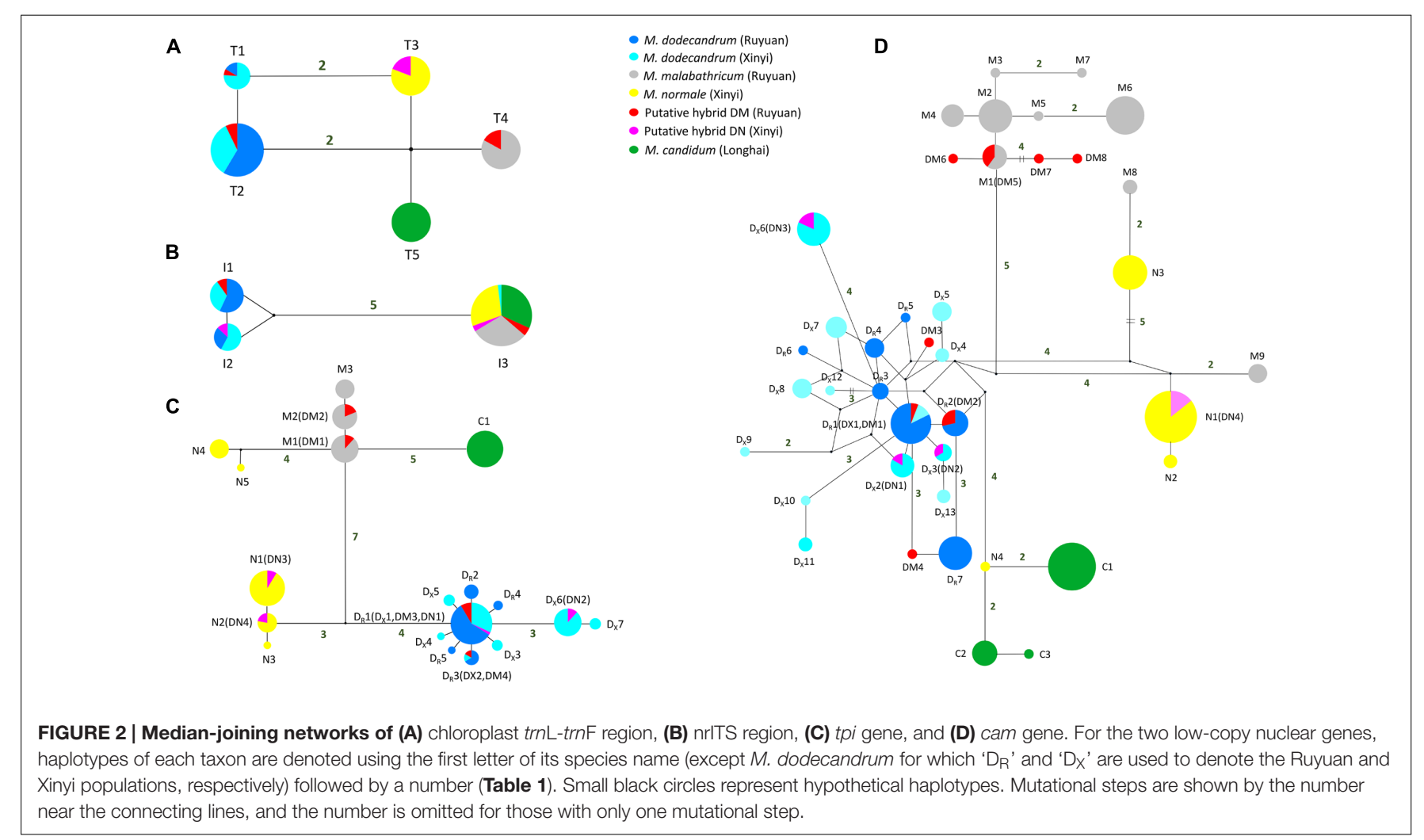

(Bandelt et al., 1999) implemented in Network $4.6^{1}$ to resolve the relationships among the haplotypes of each locus.

\section{RESULTS}

\section{Sequence Analysis of Chloroplast trnL-trnF}

The aligned length of chloroplast trnL-trnF in all samples was 825 bp. We detected four nucleotide substitutions and one

${ }^{1}$ http://www.fluxus- engineering.com insertion/deletion (indel) in these samples, which generated five haplotypes (Supplementary Table S2 and Figure 2). In each of the two populations (Ruyuan and Xinyi) of $M$. dodecandrum, we observed the same two haplotypes (T1 and T2), with one mutational step between them. For the three other species, M. malabathricum, M. normale, and M. candidum, only one unique haplotype for each species was detected. Melastoma dodecandrum, M. malabathricum, $M$. normale, and $M$. candidum can be distinguished from each other because there were no shared haplotypes between them. There were at least two mutational steps between haplotypes of any two of the putative parental species. As for DM and DN, no private haplotypes were 
found. Three of the five individuals of DM shared the haplotype with M. malabathricum, and the remaining two individuals shared the haplotypes of $M$. dodecandrum. All four individuals of DN had the same haplotype as M. normale (Supplementary Table S2). Neither DM nor DN shared haplotypes with M. candidum.

\section{Sequence Analysis of nrlTS Region}

The length of nrITS regions in the six taxa of Melastoma was $691 \mathrm{bp}$ after sequence alignment. There were five nucleotide substitutions and one 1-bp indel across these samples, which led to three haplotypes (Figure 2). No intraspecific variation was found for M. malabathricum, M. normale, and M. candidum, and they shared the same haplotype I3. Except one individual of $M$. dodecandrum from Xinyi population, which had the I3 haplotype (it should be introgressed from $M$. normale and we will discuss it later), both populations (Ruyuan and Xinyi) of M. dodecandrum had two haplotypes, I1 and I2. Only one mutational step existed between I1 and I2. There were six mutational steps between haplotype I3 and the haplotypes of M. dodecandrum (I1 and I2). All the sampled individuals of DM and DN were heterozygous at this region, with all four individuals of DM possessing $\mathrm{I} 1$ and $\mathrm{I} 2$, and all five individuals of $\mathrm{DN}$ possessing I1 and I3 (Supplementary Table S3 and Figure 2).

\section{Sequence Analysis of Two Low-Copy Nuclear Genes}

$\boldsymbol{t} \boldsymbol{p i}$ - The partial tpi gene was $703 \mathrm{bp}$ in length after sequence alignment. A total of 32 variable sites at this gene were detected. There were 11 differentially fixed sites between $M$. malabathricum and $M$. dodecandrum from Ruyuan, and on each of these sites, DM showed chromatogram signal additivity (Supplementary Table S4). Likewise, there were four sites differentially fixed in $M$. normale and $M$. dodecandrum from Xinyi, and on each of these sites, DN exhibited chromatogram signal additivity (Supplementary Table S4). In the haplotype analysis, no shared haplotypes were observed among the four species. Except for $M$. normale, haplotypes of each species formed a cluster (Figure 2). Haplotypes of $M$. normale formed two highly divergent groups. All the haplotypes detected in DM were shared with $M$. dodecandrum from Ruyuan and M. malabathricum (Figure 2 and Supplementary Table S4). Likewise, all the haplotypes found in DN were shared with either $M$. dodecandrum from Xinyi or M. normale.

cam - The aligned sequence length of the partial cam gene was $741 \mathrm{bp}$. There was only one differentially fixed site (the 70th site: $\mathrm{T}$ for $M$. dodecandrum and $\mathrm{G}$ for $M$. normale) between $M$. normale and $M$. dodecandrum from Xinyi, and on this site, DN showed chromatogram signal additivity. However, no differentially fixed sites were observed between M. malabathricum and M. dodecandrum from Ruyuan. In the haplotype analysis, $M$. dodecandrum and M. malabathricum showed more haplotypes than $M$. normale and $M$. candidum. Although $M$. malabathricum, $M$. normale, and $M$. candidum did not form their own distinct clusters, no shared haplotypes were observed between any two species. Two haplotypes of M. malabathricum (M8 and M9) were more closely related to those of $M$. normale, and one haplotype of $M$. normale (N4) was more closely related to those of $M$. candidum. All the haplotypes identified in DN were shared with $M$. dodecandrum from Xinyi and $M$. normale, while three of eight haplotypes detected in DM were shared with $M$. dodecandrum from Ruyuan and M. malabathricum. Two and three private haplotypes were closely related to those of $M$. dodecandrum and $M$. malabathricum, respectively.

\section{DISCUSSION}

\section{Molecular Evidence for Hybrid Status of the Two Semi-creeping Taxa of Melastoma}

The two semi-creeping taxa of Melastoma (DM and DN) sampled in this study resemble another previously identified hybrid M. intermedium in morphology, but we were not certain if they had the same origin or not. In this study, we obtained sequence data from chloroplast DNA and nuclear DNA to clarify their taxonomic status. At the chloroplast locus, the individuals of DM had the same haplotype as either M. malabathricum or $M$. dodecandrum, and those of DN had identical haplotype with $M$. normale only. At all three nuclear loci, each individual of DN had two haplotypes, each matching those of $M$. dodecandrum and $M$. normale (Table 2). At two of the three nuclear loci, each individual of DM had two haplotypes, each matching those of M. dodecandrum and M. malabathricum. Although DM had several private haplotypes at the cam gene, these private haplotypes were clustered together with those of $M$. dodecandrum or M. malabathricum. Considering that M. dodecandrum and M. malabathricum had relatively high levels of polymorphism at this gene, unsampled polymorphisms in the parental species are likely to have produced this pattern. Of course, we could not exclude the possibility of new mutations occurring in DM. Therefore, molecular data in our study provided compelling evidence for the hybrid status of the two semi-creeping taxa of Melastoma. The two hybrids originated from hybridization between $M$. dodecandrum and M. malabathricum (DM), and between $M$. dodecandrum and $M$. normale (DN), different from the origins of $M$. intermedium. Morphological similarity for the three hybrids is due likely to their origins from hybridization between the same creeping species, M. dodecandrum, and a different erect species, in each of the three cases. Because all the individuals of the two hybrids had two divergent haplotypes corresponding to their parental species at each of the three nuclear loci, they were all probably $\mathrm{F}_{1}$ hybrids. It is also possible that they are later-generation hybrids but more nuclear loci should be analyzed to be certain.

Previous studies have also identified natural hybridization in other combinations of Melastoma species (Dai et al., 2012; Liu et al., 2014). Along with this study, we can see that natural hybridization between species of Melastoma is relatively common. Substantial overlap in geographic distribution and flowering time, as well as shared pollinators, may facilitate the occurrence of natural hybridization in Melastoma 
(Dai et al., 2012; Liu et al., 2014). As for the focus species in our study, M. dodecandrum is distributed in southern China and northern Vietnam, while $M$. normale ranges from northern Southeast Asia to southern China. M. malabathricum is the most widespread species throughout the distribution range of Melastoma, but in China, it is confined to the northern edge of southern China. Despite the overlap in geographic distribution of each pair of parental species, we failed to find the two hybrids in many locations where their parental species overlap. The rare occurrence of DM may be due to the relatively small range overlap of its parental species. In the case of $\mathrm{DN}$, it may be caused by the difference in flowering period between its parental species. In China, M. dodecandrum flowers from May to July, and M. normale flowers usually from March to April. But in locations of high elevation like Dawuling in Xinyi, the lower temperature delays the flowering time for $M$. normale, leading to an overlap of flowering time between $M$. normale and $M$. dodecandrum. In fact, a small fraction of individuals of $M$. normale can flower in May (R. Zhou, personal observation), providing temporal opportunity for hybridization to occur. Human disturbance due to road or trail constructions may also facilitate the occurrence of hybridization between them. From our observations at the sampling sites, the two newly-discovered hybrids from Xinyi and Ruyuan, as well as the more common $M$. intermedium, were growing alongside their parental species, suggesting no niche shifts in the hybrids.

\section{The Role of Natural Hybridization in the Evolution of Melastoma}

A common outcome of interspecific hybridization is introgression. Although most introgressed loci are neutral, a few loci related to adaptation can be transferred from one species to another and thus lead to rapid adaptation of the recipient species to new environments (Baack and Rieseberg, 2007). Melastoma dodecandrum can hybridize with the three other congeneric species, however, the number of individuals for each hybrid is very small, suggesting strong reproductive isolation between $M$. dodecandrum and the three other species. Strong reproductive isolation, however, does not completely hinder interspecific introgression. One individual of $M$. dodecandrum in Xinyi population had the same nrITS haplotype (I3) as the three other species. Haplotype sharing for this individual is due more likely to introgression from the sympatric $M$. normale rather than to ancestral polymorphism in $M$. dodecandrum, as reasoned here. First, the nrITS haplotypes of 39 out of $40 \mathrm{M}$. dodecandrum individuals (I1 and I2) are highly divergent from that of the three other species (I3), with six mutational steps between them. Second, only two haplotypes (I1 and I2) were observed in all

\section{REFERENCES}

Abbott, R. J., Barton, N. H., and Good, J. M. (2016). Genomics of hybridization and its evolutionary consequences. Mol. Ecol. 25, 2325-2332. doi: 10.1111/mec. 13685

Arnold, M. L. (1997). Natural Hybridization and Evolution. New York, NY: Oxford University Press.

Arnold, M. L., and Martin, N. H. (2009). Adaptation by introgression. J. Biol. 8:82.
20 individuals of $M$. dodecandrum from Ruyuan. If the extra haplotype (I3) present in the M. dodecandrum individual had been due to ancestral polymorphism, we would expect the same haplotype to also be observed in the $M$. dodecandrum population from Ruyuan. We detected introgression to only one individual of $M$. dodecandrum from $M$. normale at only the nrITS locus, suggesting a low rate of introgression to $M$. dodecandrum from $M$. normale. Interestingly, this individual was also homozygous at the nrITS locus, which can be attributed to concerted evolution after hybridization, rapidly homogenizing the nrITS copies within a genome. Concerted evolution of the nrITS region can be observed in as early as the $\mathrm{F}_{2}$ generation (Fuertes Aguilar et al., 1999). Because the individual of $M$. dodecandrum does not have alleles of $M$. normale at other loci we studied, it must be a later-generation hybrid. Therefore, it is probable that rapid concerted evolution resulted in the homogenization of the M. normale nrITS copy in the individual. Even at a low level of introgression, adaptive alleles and corresponding adaptive traits may be transferred from one species to another, likely contributing to adaptation to various environments (Abbott et al., 2016; e.g., Lamichhaney et al., 2016). The extent of introgression and loci of adaptive introgression in Melastoma can be further explored with genomic scanning in the future.

\section{AUTHOR CONTRIBUTIONS}

RZ, QF, and YL designed the study. RZ, QF, YL, SD, PZ and ZN collected materials, and experiments were performed by $\mathrm{PZ}$ and SW. RZ, QF, YL, SD and ZN guided the experiments. PZ analyzed and interpreted the data and wrote the manuscript with guidance of RZ, WLN, and WW. All authors read and approved the final manuscript.

\section{FUNDING}

This study was supported by the Fundamental Research Funds for the Central Universities (15lgjc23), the National Natural Science Foundation of China (91231106), Guangdong Natural Science Foundation (2015A030302011), and Chang Hungta Science Foundation of Sun Yat-sen University.

\section{SUPPLEMENTARY MATERIAL}

The Supplementary Material for this article can be found online at: http://journal.frontiersin.org/article/10.3389/fpls.2017.00673/ full\#supplementary-material

Baack, E. J., and Rieseberg, L. H. (2007). A genomic view of introgression and hybrid speciation. Curr. Opin. Genet. Dev. 17, 513-518. doi: 10.1016/j.gde.2007. 09.001

Bandelt, H. J., Forster, P., and Röhl, A. (1999). Median-joining networks for inferring intraspecific phylogenies. Mol. Biol. Evol. 16, 37-48. doi: 10.1093/ oxfordjournals.molbev.a026036

Chao, L., Chen, Y., Wang, S., Liu, T., Wu, W., Dai, S., et al. (2014). One species or two? Multilocus analysis of nucleotide variation of Melastoma penicillatum and 
Melastoma sanguineum (Melastomataceae) in Hainan, China. Biochem. Syst. Ecol. 55, 275-282. doi: 10.1371/journal.pone.0096680

Dai, S., Wu, W., Zhang, R., Liu, T., Chen, Y., Shi, S., et al. (2012). Molecular evidence for hybrid origin of Melastoma intermedium. Biochem. Syst. Ecol. 41, 136-141. doi: 10.1016/j.bse.2011.12.010

Doyle, J. J., and Doyle, J. L. (1987). A rapid DNA isolation procedure for small quantities of fresh leaf tissue. Phytother. Res. 19, 11-15.

Fitzpatrick, B. M., Johnson, J. R., Kump, D. K., Smith, J. J., Voss, S. R., and Shaffer, H. B. (2010). Rapid spread of invasive genes into a threatened native species. PNAS 107, 3606-3610. doi: 10.1073/pnas.0911802107

Fuertes Aguilar, J., Rosselló, J. A., and Nieto Feliner, G. (1999). Nuclear ribosomal DNA (nrDNA) concerted evolution in natural and artificial hybrids of Armeria (Plumbaginaceae). Mol. Ecol. 8, 1341-1346. doi: 10.1046/j.1365-294X.1999. 00690.x

Goulet, B. E., Roda, F., and Hopkins, R. (2017). Hybridization in plants: old ideas, new techniques. Plant Physiol. 173, 65-78. doi: 10.1104/pp.16. 01340

Harrison, R. G., and Larson, E. L. (2014). Hybridization, introgression, and the nature of species boundaries. J. Hered. 105, 795-809. doi: 10.1093/jhered/ esu033

Lamichhaney, S., Han, F., Berglund, J., Wang, C., Almén, M. S., Webster, M. T., et al. (2016). A beak size locus in Darwin's finches facilitated character displacement during a drought. Science 352, 470-474. doi: 10.1126/science. aad8786

Librado, P., and Rozas, J. (2009). DnaSP v5: a software for comprehensive analysis of DNA polymorphism data. Bioinformatics 25, 1451-1452. doi: 10.1093/ bioinformatics/btp187

Liu, T., Chen, Y., Chao, L., Wang, S., Wu, W., Dai, S., et al. (2014). Extensive Hybridization and Introgression between Melastoma candidum and M. sanguineum. PLoS ONE 9:e96680. doi: 10.1371/journal.pone.009 6680

Meyer, K. (2001). Revision of the Southeast Asian genus Melastoma (Melastomataceae). Blumea. 46, 351-398.

Renner, S. S., and Meyer, K. (2001). Melastomeae come full circle: biogeographic reconstruction and molecular clock dating. Evolution 55, 1315-1324. doi: 10.1111/j.0014-3820.2001.tb00654.x

Rieseberg, L. H., Baird, S. J., and Desrochers, A. M. (1998). Patterns of mating in wild sunflower hybrid zones. Evolution 52, 713-726. doi: 10.2307/2411266
Taberlet, P., Gielly, L., Pautou, G., and Bouvet, J. (1991). Universal primers for amplification of three non-coding regions of chloroplast DNA. Plant Mol. Biol. 17, 1105-1109. doi: 10.1007/BF00037152

Thompson, J. D., Gibson, T. J., Plewniak, F., Jeanmougin, F., and Higgins, D. G. (1997). The CLUSTAL_X windows interface: flexible strategies for multiple sequence alignment aided by quality analysis tools. Nucleic Acids Res. 25, 4876-4882. doi: 10.1093/nar/25.24.4876

Westcott, D. A., Dennis, A. J., Bradford, M. G., Harrington, G. N., and McKeown, A. (2008). "Seed dispersal processes in Australia's tropical rainforests," in Living in a Dynamic Tropical Forest Landscape, eds N. E. Stork and S. M. Turton (Hoboken, NJ: Blackwell Publishing), 210-223.

White, T. J., Bruns, T., Lee, S., and Taylor, J. (1990). "Amplification and direct sequencing of fungal ribosomal RNA genes for phylogenies," in PCR Protocols, eds M. A. Innis, D. H. Gelfand, J. J. Sninsky, and T. J. White (San Diego, CA: Academic Press), 315-322.

Whitney, K. D., Randell, R. A., and Rieseberg, L. H. (2010). Adaptive introgression of abiotic tolerance traits in the sunflower Helianthus annuus. New Phytol. 187, 230-239. doi: 10.1111/j.1469-8137.2010.03234.x

Whittaker, R. J., and Jones, S. H. (1994). The role of frugivorous bats and birds in the rebuilding of a tropical forest ecosystem, Krakatau, Indonesia. J. Biogeogr. 21, 245-258. doi: 10.2307/2845528

Wong, K. M. (2016). The Genus Melastoma in Borneo Including 31 New Species. Sabah: Natural History Publications.

Zhang, R., Liu, T., Wu, W., Li, Y., Chao, L., Huang, L., et al. (2013). Molecular evidence for natural hybridization in the mangrove fern genus Acrostichum. BMC Plant Biol. 13:74. doi: 10.1186/1471-2229-13-74

Conflict of Interest Statement: The authors declare that the research was conducted in the absence of any commercial or financial relationships that could be construed as a potential conflict of interest.

Copyright (c) 2017 Zou, Ng, Wu, Dai, Ning, Wang, Liu, Fan and Zhou. This is an open-access article distributed under the terms of the Creative Commons Attribution License (CC BY). The use, distribution or reproduction in other forums is permitted, provided the original author(s) or licensor are credited and that the original publication in this journal is cited, in accordance with accepted academic practice. No use, distribution or reproduction is permitted which does not comply with these terms. 